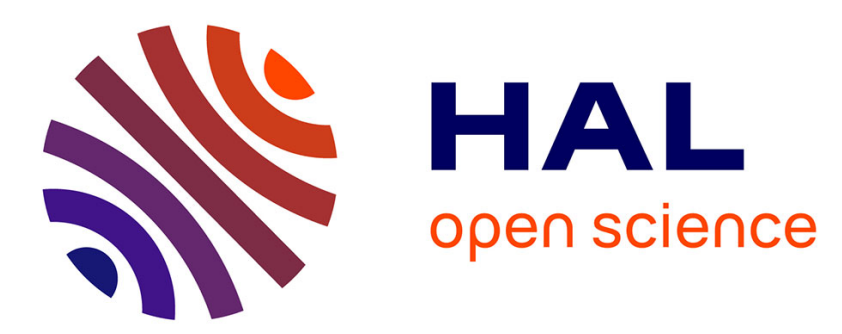

\title{
Photothermal Determination of Infrared Emissivity of Selective Solar Absorbing Coatings
}

J. D. Macias, Jose Ordonez-Miranda, F. I. Lizama-Tzec, Oscar Arès, Jose Bante-Guerra, Gerko Oskam, Romeo de Coss, Juan Jose Alvarado-Gil

\section{- To cite this version:}

J. D. Macias, Jose Ordonez-Miranda, F. I. Lizama-Tzec, Oscar Arès, Jose Bante-Guerra, et al.. Photothermal Determination of Infrared Emissivity of Selective Solar Absorbing Coatings. International Journal of Thermophysics, 2014, 36 (5), pp.1051-1056. 10.1007/s10765-014-1614-4 . hal-01357616

\section{HAL Id: hal-01357616 https://hal.science/hal-01357616}

Submitted on 1 Sep 2016

HAL is a multi-disciplinary open access archive for the deposit and dissemination of scientific research documents, whether they are published or not. The documents may come from teaching and research institutions in France or abroad, or from public or private research centers.
L'archive ouverte pluridisciplinaire HAL, est destinée au dépôt et à la diffusion de documents scientifiques de niveau recherche, publiés ou non, émanant des établissements d'enseignement et de recherche français ou étrangers, des laboratoires publics ou privés. 


\section{Photothermal Determination of Infrared Emissivity of Selective Solar Absorbing Coatings}

J. D. Macias ${ }^{1}$, J. Ordonez-Miranda ${ }^{2}$, I. Lizama-Tzec ${ }^{1}$, O. Arés-Muzio ${ }^{1}$, J. BanteGuerra $^{1}$, G. Oskam ${ }^{1}$, R. de Coss ${ }^{1}$ and J.J. Alvarado-Gil1,3

${ }^{1}$ Departamento de Física Aplicada, Centro de Investigación y de Estudios Avanzados del IPN-Unidad Mérida, Carretera Antigua a Progreso km. 6, A.P. 73 Cordemex, Mérida, Yucatán, 97310, México.

${ }^{2}$ Laboratoire d'Énergétique Moléculaire et Macroscopique, Combustion, UPR CNRS 288, Ecole Centrale Paris, Grande Voie des Vignes, 92295 Chatenay Malabry, France.

${ }^{3}$ To whom correspondence should be addressed. E-mail: jjag@mda.cinvestav.mx 


\begin{abstract}
The Infrared emission of glassy carbon, stainless steel and stainless steel with a selective coating of $\mathrm{NiNiO}$ has been using the thermal-wave resonant cavity heated up with a modulated laser beam. This is achieved performing a length scan of the cavity at a fixed frequency of $5 \mathrm{~Hz}$. It is observed experimentally that: 1) the mechanisms of heat conduction and radiation co-exist inside the cavity, through the coupling of the dc and ac components of the temperature. 2) The radiation effect shows up in both the amplitude and phase signals for cavity thicknesses greater than the diffusion length of the intra-cavity air. Using a suitable theoretical model the experimental data for the amplitude and phase has allowed the determination of the infrared emissivity of the studied materials.
\end{abstract}

Keywords: Infrared Emissivity; Heat radiation; Solar absorbing coatings; Thermal wave resonant cavity 


\section{Introduction}

The thermal wave resonant cavity (TWRC) is one of the most reliable and accurate photothermal techniques to measure the thermal diffusivity of fluids [1]. It was first introduced in 1995 by Shen and Mandelis [2], who measured the thermal diffusivity of air at $294 \mathrm{~K}$ with three significant figures. By performing a length scan of an air cavity, Shen et al. [3] found that as the cavity thickness increases, the recorded phase of the photothermal signal does not behave according to the theoretical predictions of the pure heat conduction. The obtained experimental behavior for the phase was theoretically explained by considering simultaneously the conduction and radiation as the two mechanisms responsible for the photothermal heating of the cavity. By using a $\mathrm{Cr}-\mathrm{Ni}$ thin film as a resistive heating strip operating with dc and ac currents, the radiation effect appearing in the phase was used to determine the infrared emissivity of the film [3]. More recently, Ordonez-Miranda et al. [4] developed the theoretical formalism for describing the heat conduction and radiation mechanisms in a TWRC heated up with a modulated laser beam. They showed that the coupling between the $\mathrm{dc}$ and ac components of the temperature could be used to determine the emissivity of the illuminated layer by means of various simple expressions. In particular this latter formalism can be used to determine the emissivity of the coating of selective solar absorbers. These devices have high efficiency to convert the incident solar radiation into useful heat, and they usually consist of a coating with high solar absorptance, deposited on a substrate of low thermal emittance to minimize thermal back-radiation [5]. The efficiency of a selective solar absorber can be much higher than the one of a nonselective one [7], and it depends strongly on the optical and thermal properties of its coating and substrate. The thermal characterization of these components is therefore critical, and it can be done through the determination of their emissivity.

In this work, the experimental measurement of the emissivity of selective solar absorbing coatings is reported. This is performed varying the thickness of the intracavity air of a TWRC excited with a modulated laser beam at fixed modulation frequency, and fitting the experimental values for the amplitude and phase using a previously developed theoretical model [4]. In both components of the photothermal signal, amplitude and phase, the contribution of the radiation can be observed experimentallv in agreement with effects predicted theoreticallv. 


\section{Experimental Apparatus and Procedures}

\subsection{Samples}

Samples of glassy carbon (50 $\mu$ m-thick), stainless steel substrate (AISI 316, $50 \mu \mathrm{m}$ thick) and stainless steel substrate with a selective coating have been used as a heater layer (see Fig. 1). This coating of $\mathrm{NiNiO}$ has been deposited with an electrochemical deposition method [5]. The coating consists of a $1 \mu$ m-thick layer of nickel (high thermal reflectance layer) in thermal contact with a $200 \mathrm{~nm}$-thick of nickel oxide (high solar absorbance layer) as detailed in Fig. 1.

\subsection{Description of the Experimental Technique}

\subsubsection{Principle of the Technique}

The working principle of the TWRC is based on placing the fluid sample in between of two parallel walls: the first one is a thin foil, which is usually heated up with a modulated laser beam and the second wall is the sensor (usually of pyroelectric), which detects the amplitude and phase of the photothermal signal as a voltage. These signals are then compared to a proper theoretical model to evaluate the thermal diffusivity of the intra-cavity fluid and/or the emissivity of the thin foil.

\subsubsection{Apparatus}

The experimental set up for the TWRC used in this work is similar those describe in literature[2,6,7], as shown in Fig. 1. The thermal waves are generated by illuminating the heater with a solid-state laser $(500 \mathrm{~mW}, 670 \mathrm{~nm})$ and the length of the intra-cavity air between the pyroelectric sensor and the heater is slowly varied in steps of $20 \mu \mathrm{m}$ through a stepping motor attached to the heater. The signals detected by the sensor are then amplified with a low-noise preamplifier (SR-560), processed by a lock-in amplifier (SR-830 DSP), and displayed in a personal computer. These latter three devices are the components of the block of data acquisition and control shown in Fig. 1.

\subsection{Experimental Procedures}

To optimize the signal/noise ratio, the modulation frequency of the laser beam exciting the TWRC has been fixed at $5 \mathrm{~Hz}$. Under these conditions the three heater samples are in the thermally thin regime; while the intra-cavity air is thermally thick [8]. For these regimes of the heater and cavity, the emissivity of the samples can be determined through a simple theoretical model $\lceil 41$. Bv performing a length scan of the cavitv. the 
experimental data for the amplitude and phase detected by the pyroelectric sensor have been recorded for the three samples. Using the normalized amplitude and phase, the emissivity $(\in)$ of the inner (non-illuminated) surface of each sample has been determined. The normalized pyroelectric signal $V(f)$ at the modulation frequency $f$ is given by [4]:

$$
V(f)=\frac{\varepsilon_{2}}{\rho_{1} c_{1} l_{1} \sqrt{2 \pi f}}\left(\frac{1}{\sqrt{i} \sinh \left(\sigma_{2} l_{2}\right)}-i \frac{\epsilon}{\epsilon_{0}}\right),
$$

where $\rho_{1}, c_{1}$, and $l_{1}$ are the density, specific heat capacity, and thickness of the heater, respectively; $\sigma_{2}=(1+i) / \mu_{2}$ and $\epsilon_{0}=\varepsilon_{2} \sqrt{2 \pi f} /\left(4 \sigma B_{2}^{3}\right)$. Here $\mu_{2}=\sqrt{\alpha_{2} / \pi f}$ is the thermal diffusion length, $\varepsilon_{2}$ is the thermal effusivity, and $\alpha_{2}$ is the thermal diffusivity of the air inside the cavity; $B_{2}$ is the dc temperature of the inner surface of the heater and $\sigma=5.67 \times 10^{-8} \mathrm{Wm}^{-2} \mathrm{~K}^{-4}$ is the Stefan-Boltzmann constant. Note that the first term in the parenthesis of Eq. (1), is the typical contribution due to heat conduction, while the second one stands for the radiation contribution, which only affects to the imaginary part of $V(f)$.

\section{Results}

The experimental data for the normalized amplitude $A$ and phase $\phi$ as a function of the normalized thickness $l_{2} / \mu_{2}$ of the cavity are shown in Figs. 2(a) and 2(b), respectively. Here $l_{2}$ is the cavity thickness and $\mu_{2}=1.19 \mathrm{~mm}$ is the thermal diffusion length of the intra-cavity air. Note that the normalized amplitude of the three samples exhibits a pronounced dip, and along with the normalized phase tend to values which are independent of cavity length, as $l_{2} / \mu_{2}$ increases. The deeper the dip of the amplitude $(A)$, the stronger the radiation contribution to the modulated heating of the TWRC. This indicates that the emissivity of glassy carbon is expected to be higher than that of steel. These features represent the signature of the radiation effect on $A$ and $\phi$ [4], and therefore they can be used to determine the emissivity of each sample, through a fit of the experimental data with a theoretical model shown in Eq. (1). The fitting parameter of this model is the emissivity of the inner surface of the sample, provided that its dc temperature $\left(36^{\circ} \mathrm{C}\right.$ in our experiment) is known. The solid lines in Figs. 2(a) and 2(b) represent the results of this fit, which are in fairly good agreement with the corresponding experimental data. For the case of the normalized amplitude. the fit has 
been performed around the dip only, because of the simple model describing its behavior [4]. The results of this fitting procedure on the normalized amplitude and phase are summarized in Table I. Note that the emissivity values provided by the amplitude and phase are consistent between them and agree with those reported in the literature, however the emissivity obtained by means of the normalized amplitude is closer to the literature value, than that provided by the phase. This is reasonable, given that the signal/noise ratio is higher for small cavity thicknesses and because the fit of the amplitude has been performed for normalized cavity thicknesses $\left(l_{2} / \mu_{2}\right)$ smaller than the ones used for the phase. Both the amplitude and phase have been fitted in the range of cavity thicknesses where the signature of the radiation effect is present. This effect appears at different cavity thicknesses for the amplitude and phase, as was shown previously [4]. It is also clear that the amplitude curve exhibits larger changes in curvature as a function of the frequency, and a higher sensitivity to radiation effects that make it more suitable to determine the emissivity. These results show that the presented experimental methodology can be applied to perform the measurement of the emissivity of layered materials. For those materials with low emissivity, the radiation effect on the amplitude and phase can be enhanced performing the required experiments in vacuum conditions.

\section{Conclusions}

Experimental measurement of the emissivity of glassy carbon, stainless steel and stainless steel with a selective coating of $\mathrm{NiNiO}$ has been performed by means of a thermal-wave resonant cavity heated up with a modulated laser beam. This has been achieved varying the thickness of the intra-cavity air excited with a fixed modulation frequency of $5 \mathrm{~Hz}$, and fitting the obtained experimental data for the amplitude and phase to a suitable theoretical model. The radiation effect has been observed in both the amplitude and phase of the photothermal signal, for cavity thicknesses greater than the diffusion length of air, where the heat conduction is weak. The emissivity values provided by the amplitude and phase are consistent between them and they are in good agreement with data reported in the literature, for each sample. The measurements based on the amplitude provide higher accuracy than those arising from the phase through relatively smaller cavity thicknesses. The developed experimental methodology, along 
with the obtained results, show that the thermal-wave resonant cavity can be used to determine the emissivity of this kind of layered materials.

\section{Acknowledgments}

This work was supported by CONACYT under the FORDECYT program and grant number 174532. The authors are grateful to Rosemberg Oswaldo Rodriguez Salas for his valuable technical support. 


\section{References}

1. A. Matvienko, A. Mandelis, Rev. Sci. Instrum. 77, 064906 (2006)

2. J. Shen, A. Mandelis, Rev. Sci. Instrum. 66, 4999 (1995)

3. J. Shen, A. Mandelis, H. Tsai, Rev. Sci. Instrum. 69, 197 (1998)

4. J. Ordonez-Miranda, J. J. Alvarado-Gil, Int. J. Therm. Sci. 74, 208 (2013)

5. M. Lira-Cantú, A. Morales Sabio, A. Brustenga, P. Gómez-Romero, Sol. Energy Mater. Sol. Cells, 87, 685 (2005)

6. J. A. Balderas-López, A. Mandelis, J. A. Garcia, Rev. Sci. Instrum. 71, 2933 (2000)

7. J. A. Balderas-López, Rev. Sci. Instrum. 82, 074905 (2011)

8. A. Rosencwaig, J. Appl. Phys. 47, 64 (1976)

9. M. Balat-Pichelin, J. F. Robert, J. L. Sans, Appl. Surf. Sci. 253, 778 (2006)

10. H. Ryuto, H. Hasebe, N. Fukunishi, S. Yokouchi, A. Goto, M. Kase, Y. Yano, Nucl. Instruments Methods Phys. Res. Sect. A 569, 697 (2006)

11. A. Baldwin, J. Lovell-Smith, J. Food Eng., 17, 1 (1992)

12. J. Lienhard, A heat transfer textbook, (Dover publications, New York 2011)

13. A. Othonos, M. Nestoros, D. Palmerio, C. Christofides, R. S. Bes, J. P. Traverse, Sol. Energy Mater. Sol. Cells, 51, 171 (1998) 
Table 1 Infrared emissivity of the studied samples.

\begin{tabular}{|c|c|c|c|}
\hline \multirow{2}{*}{ Samples } & \multicolumn{3}{|c|}{ Emissivity } \\
\cline { 2 - 4 } & Amplitude & Phase & Literature \\
\hline Glassy carbon & $0.568 \pm 0.028$ & $0.446 \pm 0.045$ & $0.60[9]$ \\
& & & $0.65[10]$ \\
\hline Steel & $0.253 \pm 0.013$ & $0.362 \pm 0.037$ & $0.28[11]$ \\
& & & $0.35[12]$ \\
\hline Steel+NiNiO & $0.199 \pm 0.009$ & $0.283 \pm 0.028$ & $0.17[13]$ \\
\hline
\end{tabular}




\section{Figure Captions}

Fig. 1 Experimental setup of the thermal wave cavity used in this work.

Fig. 2 Normalized (a) amplitude and (b) phase of the temperature field as a function of the normalized cavity thickness for three samples glassy carbon, steel and steel with a coating of NiNiO. The solid lines stand for the best fit using a theoretical model [4]. 
Fig. 1

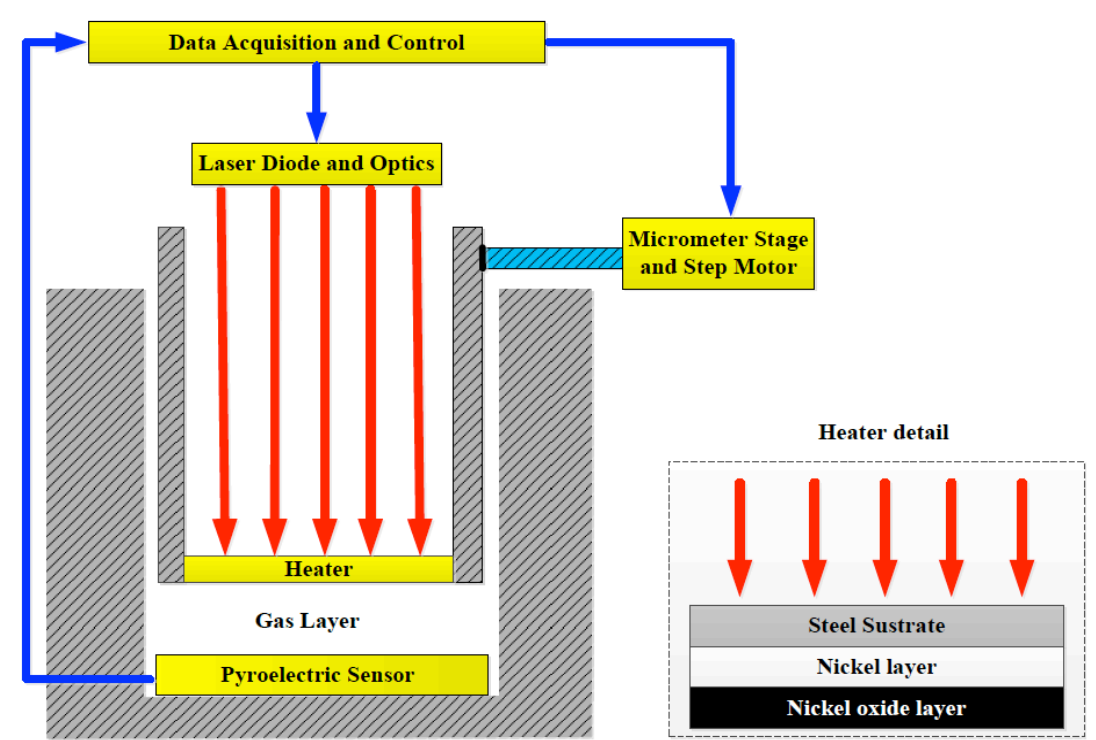


Fig. 2

(a)

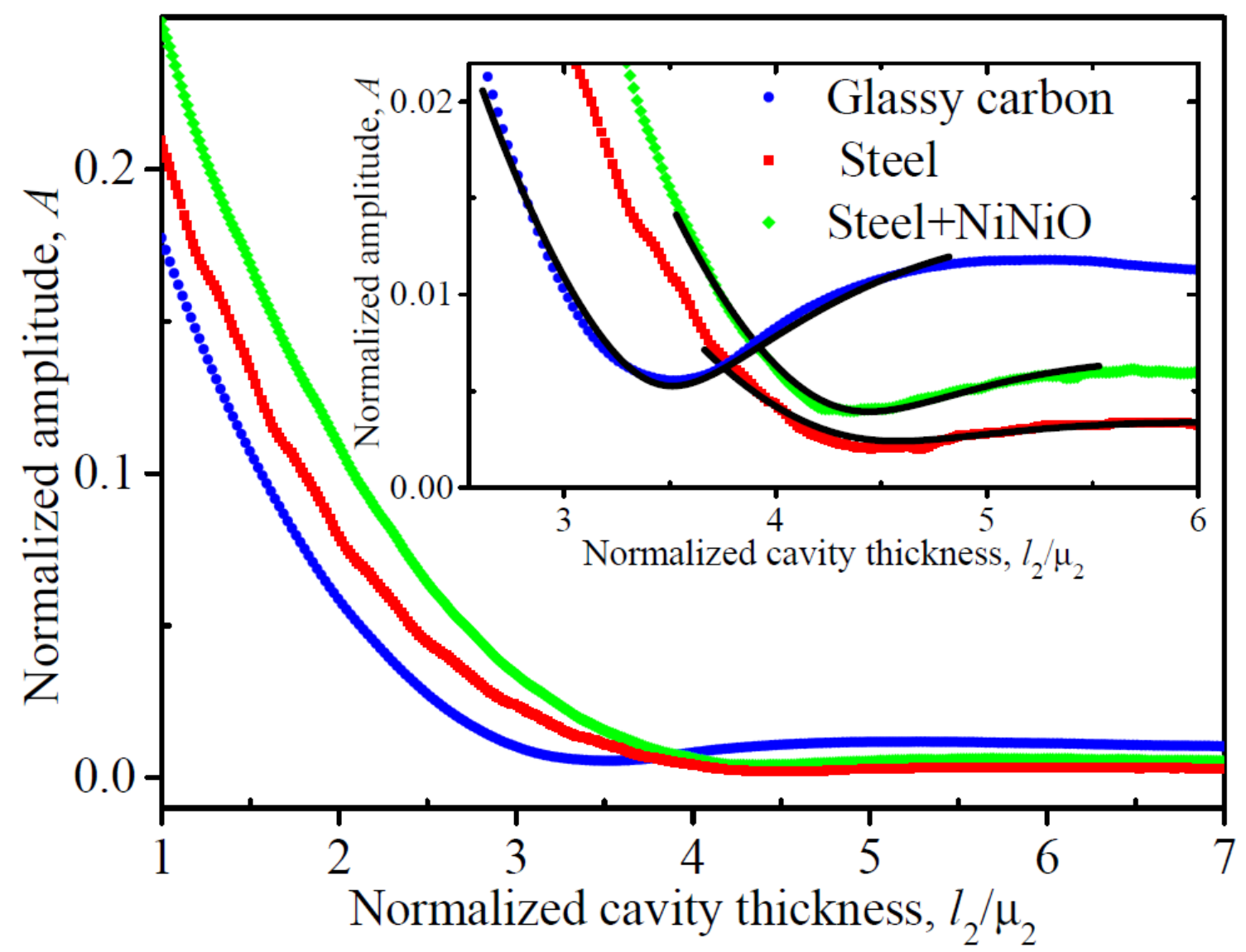


Fig. 2

(b)

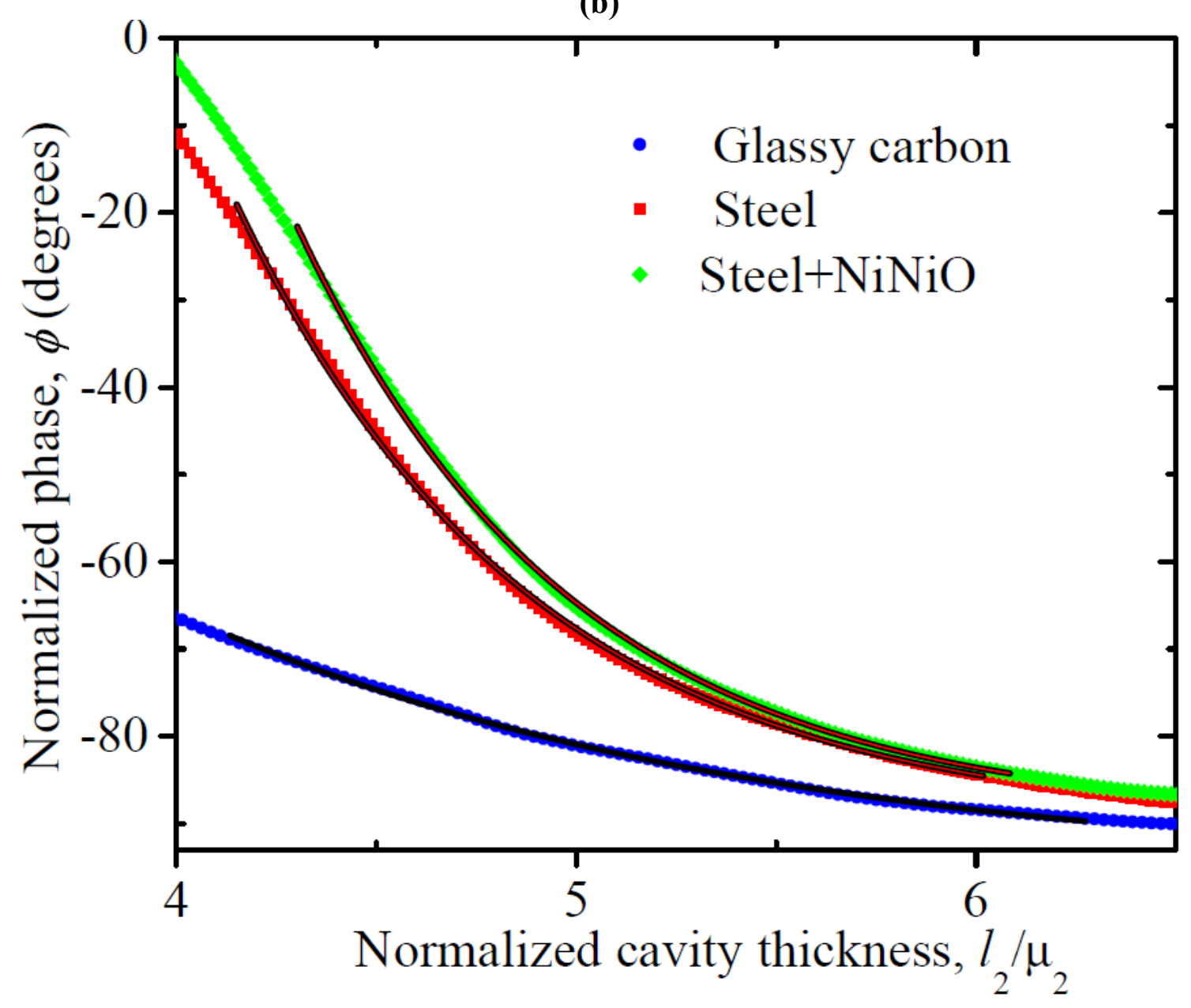

